\title{
Changes in fatal and near-fatal asthma in an urban area of South Africa from 1980-1997
}

\author{
H.J. Zar*, D. Stickells", A. Toerien*, D. Wilson\#, M. Klein*, E.D. Bateman\#
}

Changes in fatal and near-fatal asthma in an urban area of South Africa from $1980-$ 1997. H.J. Zar, D. Stickells, A. Toerien, D. Wilson, M. Klein, E.D. Bateman. (C) RS Journals Ltd 2001.

ABSTRACT: Over the past two decades, changes in the health services designed to improve access to and quality of asthma care have occurred in South Africa. The aim of this study was to investigate the incidence of fatal and near-fatal asthma (NFA) from 1980 - 1997 in an urban part of South Africa.

A retrospective analysis of fatal asthma and NFA in the Cape Town City Council area was performed. Mortality data were obtained from death notification records. Data on NFA were obtained from the records of patients admitted for asthma to intensive care units (ICUs) at major academic hospitals serving the area.

There were 1,506 deaths (mean age 56 yrs) from asthma reported; 39 and 3\% occurred in people under 55 and 15 yrs, respectively. Average annual asthma mortality rate $(8.1 \pm 1.9$ per 100,000 population) was highest amongst people of mixed race $(10.1 \pm 2.0)$, followed by Blacks $(6.8 \pm 3.1)$ and Whites $(5.0 \pm 1.9, \mathrm{p}<0.001)$. Asthma mortality declined by 0.28 deaths per 100,000 population per year; rates decreased in all ethnic groups. Most deaths $(\mathbf{7 2 . 3} \%)$ were outside a health facility and a higher number of deaths occurred on weekends $\left(223\right.$ deaths $\left.^{\prime} d a y^{-1}\right)$ than weekdays $\left(207\right.$ deaths $^{-1}$ day $^{-1}$, $\mathrm{p}=0.014)$. Paediatric asthma ICU admissions declined by 1.81 children $\cdot \mathrm{yr}^{-1}$. The annual number of adults admitted to ICU for asthma, and the proportion requiring intermittent positive pressure ventilation did not change.

These results indicate that the incidence of fatal and near-fatal asthma in this area has declined over the period and this may reflect improved asthma management. However, the relatively high asthma mortality rate in people of mixed race and the predominance of deaths outside health facilities and on weekends suggest problems with access to care.

Eur Respir J 2001; 18: 33-37.
*Dept of Paediatrics and Child Health and ${ }^{\#}$ Respiratory Clinic, Dept of Medicine, University of Cape Town, Cape Town, South Africa.

Correspondence: H.J. Zar

Child Health Unit

Dept of Paediatrics

University of Cape Town

46 Sawkins Road

Rondebosch 7700

South Africa

Fax: 27216895403

Keywords: Mortality

near-fatal asthma

South Africa

Received: September 182000

Accepted after revision March 42001

This study was supported by The Medical Research Council, South Africa and 3M Pharmaceuticals.
The incidence of asthma worldwide has increased despite advances in the understanding of the pathophysiology of the disease, emphasis on early use of prophylactic corticosteroids, improved methods of drug delivery, and standardization of management guidelines [1, 2]. The impact of improvements in asthma care can be evaluated by examining the trends in utilization of acute care facilities for exacerbations and the incidence of asthma deaths.

There are few data on asthma mortality in South Africa. A study of asthma deaths in Cape Town during 1980-1982 reported an annual mortality of 8 per 100,000 population, which was three times that of the UK [3]. A more recent study of national asthma mortality 1962-1988 described South Africans of mixed race as having one of the highest rates of death from asthma in the world, without evidence of decline over this period [4]. However, over the past two decades, important changes in health services designed to improve access to and quality of asthma management and education have occurred in South Africa. Primary health facilities including designated asthma clinics have been developed. A national asthma education programme has been established and management guidelines produced, which advocate early use of inhaled corticosteroids [5]. The dismantling of apartheid in 1994 has allowed for greater equity of healthcare for all ethnic groups. Such changes may have impacted on asthma morbidity and mortality.

Severe life threatening asthma (near-fatal asthma (NFA)) is an important risk factor for subsequent mortality from asthma [2, 6]. The incidence of fatal asthma and NFA attacks can therefore be used to reflect the quality and accessibility of asthma care. This study examines the trends in the incidence of fatal asthma and NFA amongst adults and children in an urban area of South Africa, from 1980-1997.

\section{Methods}

\section{Study design}

A retrospective analysis of the incidence of fatal asthma and NFA in the Cape Town City Council area 
was performed from $1980-1997$, inclusive. This is a rapidly growing urban area of South Africa whose population comprises three main ethnic groups; people of mixed race, African (Black) and European descent (White). The source of data was death notifications and records of intensive care unit (ICU) admissions for asthma over this period.

\section{Methods}

Mortality data were obtained from the death notification records of the Medical Officer of Health of the Cape Town City Council, in which asthma was listed as the sole cause of death or together with a secondary diagnosis of respiratory failure or cardiorespiratory arrest. Data were also analysed for children ( $<15$ yrs) and for subjects $(<55 \mathrm{yrs})$ in whom misclassification of asthma as the cause of death is less likely to occur. These age categories were selected as they are the age groups used in the published population data. Population data were obtained from the annual reports of the Medical Officer of Health for the City of Cape Town including population number, ethnicity, sex and age distribution [7]. More detailed agespecific population data were available from the South African Census data for the years 1985, 1991 and 1996; age-specific mortality rates were therefore calculated for these years. A single category of age group 5-34 yrs was used, as miscoding of asthma as a cause of death in this age group is low.

The incidence of NFA was obtained from asthma admissions to the ICUs of academic hospitals associated with the University of Cape Town; Groote Schuur Hospital (GSH) and Red Cross War Memorial Children's Hospital (RXH) for 1980-1997, inclusive. These two hospitals are the major referral hospitals in the Cape Town City Council area that have ICU beds. Information was obtained from the ICU records of patients admitted with a primary diagnosis of asthma. The study was designed to investigate the epidemiology of deaths and ICU admissions due to asthma. Patients admitted to ICUs represent those with lifethreatening attacks, a proportion of whom might have died had this intervention not been available.

\section{Analysis}

Mortality data were analysed by age, ethnic group, place of death (at home or at a health facility), day of death (weekend or weekday) and month (to assess the impact of season). Analysis of data by ethnic group was necessary to permit comparisons with prior reports, as the health system was previously organized along ethnic lines (under an apartheid system). In addition, ethnicity in South Africa has served as a strong indicator of socioeconomic status. Values were expressed as mean $\pm \mathrm{SD}$. Chi-squared tests were used for categorical variables and unpaired t-tests for continuous variables. Tests based on the Poisson distribution were used to compare the incidence of deaths on weekdays with that on weekends. Regression analysis was used to investigate the change in asthma mortality or ICU admissions over time, as well as the relationship between this change in mortality and ethnicity. The slope of the regression line (coefficient of the time variable) was used as the estimate of annual change in mortality or in number of ICU admissions.

\section{Results}

\section{Mortality from asthma}

During the 18-yr period, 1,506 deaths from asthma were recorded of which $582(38.6 \%)$ were in people $<55$ yrs and $49(3.3 \%)$ in children $<15$ yrs (fig. 1$)$. In the age group $5-34$ yrs, there were 157 (10.4\%) deaths. The mean age of subjects who died was 56 (range $1-87$ ) yrs. Males accounted for $736(48.9 \%)$ of those dying and mean \pm SD annual asthma mortality rate was lower in males than females $(7.3 \pm 1.67$ per 100,000 and $8.4 \pm 2.44$ per 100,000 females, respectively, relative risk (RR) $0.88(0.79-0.99), p=0.03)$. The annual population growth for this area was $2.46 \%$.

The average annual asthma mortality rate was $8.1 \pm 1.9$ per 100,000 population. Overall, asthma mortality declined by 0.28 deaths per 100,000 population per year; the majority of this decline $(0.23$ deaths per 100,000 population per year) occurred in $<55$ yrs of age (fig. 1, table 1). Age-specific mortality rates for the years 1985, 1991 and 1996 were 0 for the $0-4$ yrs group; $3.0,1.5$ and 1.5 for the $5-34$ yrs group; and 18.5, 10.7 and 9.7 for the $35-54$ yrs group. A decrease in asthma mortality was found in all ethnic groups $(\mathrm{p}<0.001$, fig. 2$)$ and the annual rate of decline in asthma mortality did not differ significantly by ethnic group $(p=0.14$ for the difference between Whites and Blacks, $\mathrm{p}=0.221$ for the difference between mixed race and Blacks). More deaths (71\%) occurred in people of mixed race compared to Whites

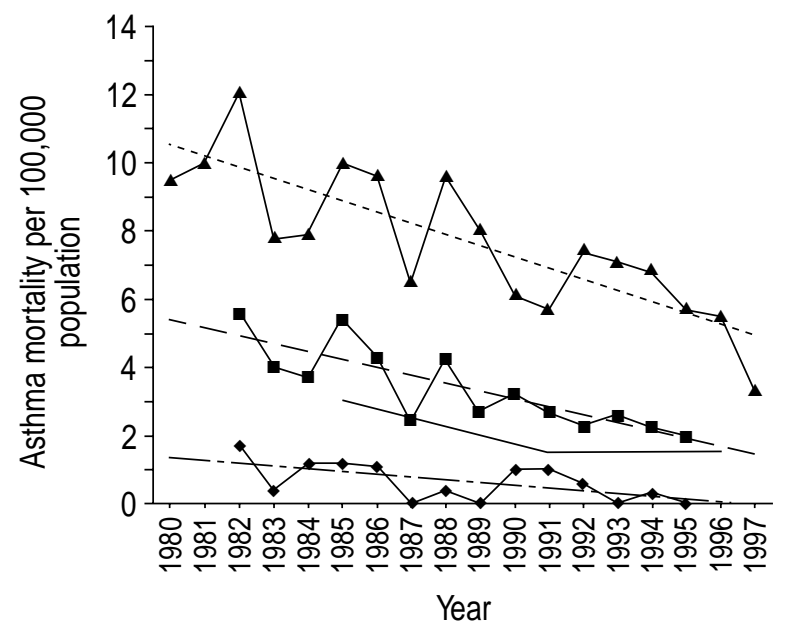

Fig. 1. - Age-specific asthma mortality rates for 1980-1997. $\boldsymbol{\Lambda}$ all subjects; - - - - - linear regression, $\mathrm{R}^{2}=0.6556$ for all subjects; $\square$ : subjects $<55$ yrs; - - - : linear regression, $\mathrm{R}^{2}=0.6768$ for subjects $<55$ yrs; $\longrightarrow$ : subjects $5-34$ yrs; $\bullet$ : subjects $<15$ yrs; $--\cdots$ : linear regression, $\mathrm{R}^{2}=0.35$ for subjects $<15$ yrs. 
Table 1.-Decline in asthma mortality by age and ethnic group, 1980-1997

\begin{tabular}{lcll}
\hline Subjects & Change in mortality* & $95 \%$ CI & p-value \\
\hline All & -0.28 & $-0.43--0.15$ & 0.001 \\
$<55$ yrs & -0.23 & $-0.55-0.09$ & 0.003 \\
$5-34$ yrs** & -0.13 & $-0.30-0.04$ & 0.381 \\
$<15$ yrs & -0.08 & $-0.14--0.02$ & 0.026 \\
Mixed race & -0.28 & $-0.45--0.11$ & 0.007 \\
Black & -0.45 & $-0.69--0.21$ & 0.002 \\
White & -0.24 & $-0.41--0.07$ & 0.017 \\
\hline
\end{tabular}

CI: confidence interval; *: expressed as number of deaths per 100,000 population per yr; $* *$ : calculated for 1980,1991 and 1996, as population data for this age group were available only for these years.

$(16 \%)$ and Blacks $(13 \% ; \mathrm{p}<0.001)$. The average annual asthma mortality rate was $10.1 \pm 2.0$ amongst mixed race people, $6.8 \pm 3.1$ in Blacks and $5 \pm 1.9$ in Whites.

There was a seasonal variation in asthma deaths. For all subjects, more deaths occurred in winter (32\%) (June to August, inclusive) compared to autumn $(25 \%)$, spring $(24 \%)$ or summer $(19 \%), \mathrm{p}<0.001$. There was no predominant seasonal distribution of asthma mortality for those aged $5-34$ yrs $(21 \%$ deaths in winter, $32 \%$ in autumn, $24 \%$ in spring and $23 \%$ in summer, $\mathrm{p}=0.154)$ or $35-54$ yrs $(28 \%$ deaths in winter, $25 \%$ in autumn, $26 \%$ in spring and $21 \%$ in summer, $\mathrm{p}=0.07)$. Overall for those $<55 \mathrm{yrs}, 26 \%$ of deaths occurred in winter compared to $28 \%$ in autumn, as well as $26 \%$ in spring and $20 \%$ in summer (fig. 3).

The majority $(72.3 \%)$ of asthma deaths occurred outside a hospital facility. Amongst the subgroup of people $<55 \mathrm{yrs}, 66.7 \%$ of asthma deaths occurred at home. In the age group 5-34 yrs, $82(52.2 \%)$ deaths occurred outside a health facility. Of the deaths outside a health facility, $74 \%$ occurred in mixed race people, $14 \%$ in Whites and $12 \%$ in Blacks. The proportion of deaths occurring outside a health facility differed by ethnic group, with $70 \%$ of all

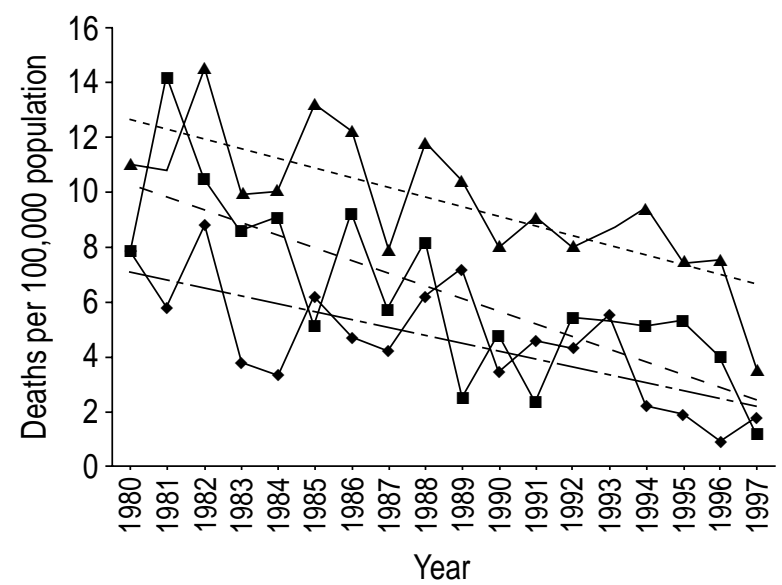

Fig. 2. - Asthma mortality rates by ethnic group for 1980-1997. $\boldsymbol{\Delta}$ : mixed race subjects; - - - - - l linear regression, $\mathrm{R}^{2}=0.5505$ for mixed race subjects; $\square$ : Blacks; --- : linear regression, $\mathrm{R}^{2}=0.5792$ for Blacks; $\rightarrow$ : White; ---: linear regression, $\mathrm{R}^{2}=0.4954$ for Whites.

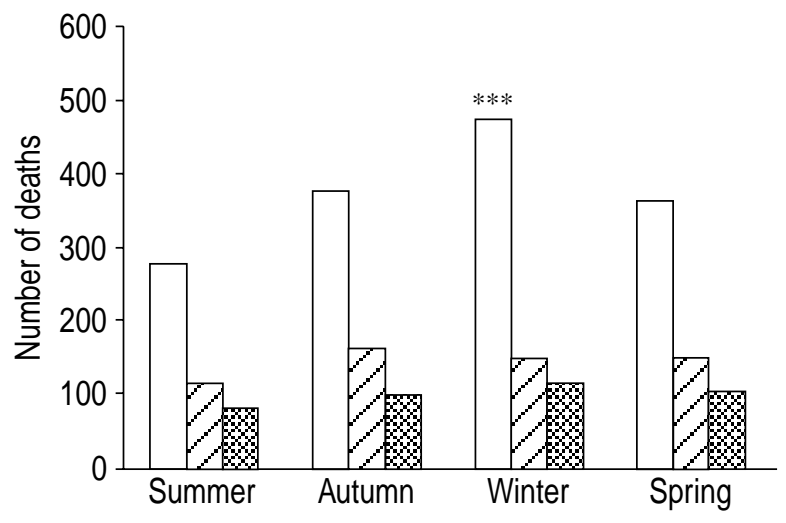

Fig. 3. - Seasonal distribution of deaths for $1980-1997$. $\square$ : all subjects; $\square$ : subjects $<55$ yrs; : subjects $5-34$ yrs. $* * *: p<0.001$ compared to other seasons.

mixed race deaths, $66 \%$ of deaths in Black people and $60 \%$ of deaths amongst Whites occurring at home $(p=0.014)$. Of the deaths occurring at health facilities other than ICUs, $50 \%$ were at tertiary institutions where ICU care was provided, $31 \%$ at secondary hospitals without ICU facilities, $11 \%$ at day hospitals (outpatient facilities only) and 8\% at clinics. An increased number of deaths occurred on weekends $\left(223 \cdot \mathrm{day}^{-1}\right)$ compared to weekdays $\left(207 \cdot\right.$ day $^{-1}$; Poisson $\mathrm{p}=0.014)$. This difference was also found in those who died outside health facilities (165 deaths weekend day $^{-1}$ compared to 148 deaths $\cdot$ weekday $^{-1}$; Poisson $\mathrm{p}=0.012)$.

\section{Near-fatal asthma attacks}

Over the 18 yrs, 487 children with a mean age of 5.8 yrs (range 7 months -15.5 yrs) were admitted to RXH ICU for treatment of acute, severe asthma. Paediatric admissions to ICUs declined over this period (fig. 4); the average change in admissions was -1.81 children $\cdot$ year $^{-1}(95 \%$ confidence interval (CI) $-3.03-0.59, p=0.01)$. Intermittent positive pressure

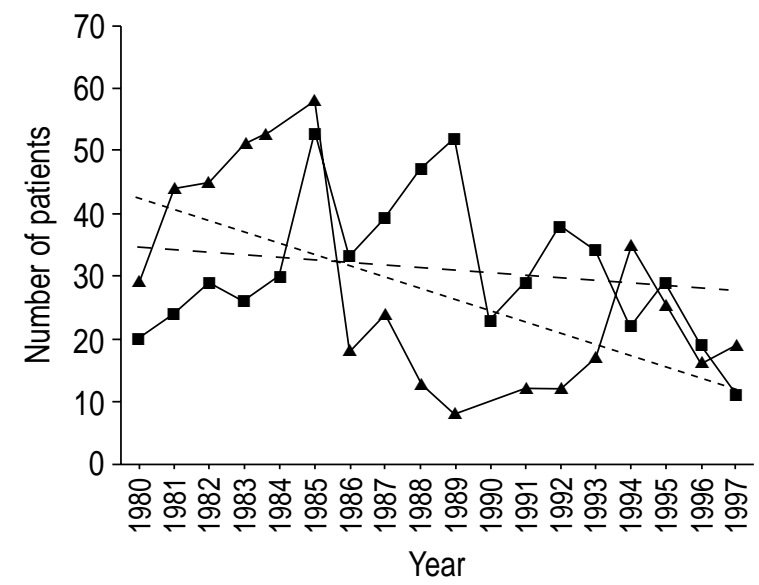

Fig. 4. - Asthma admissions to intensive care units for 1980 1997. A : paediatric admission; - - - - - linear regression, $\mathrm{R}^{2}=0.3451$ for paediatric admissions; $\mathbf{\square}$ : adults admission; - - : linear regression, $\mathrm{R}^{2}=0.0382$ for adults admission 
ventilation (IPPV) was required by 37 (7.6\%) children and the mean annual rate of ventilation of $8.5 \pm 4.9 \%$ did not change significantly over the $18 \mathrm{yrs}$. The inhospital mortality rate of ICU patients with asthma was $1 \%$.

During the same period, 558 adults (mean age 39 (range 16.6-81) yrs) were admitted to GSH ICU for asthma. The majority of patients $(74 \%)$ were of mixed race, while White and Black patients accounted for $13 \%$ of admissions each. In all ethnic groups, female patients predominated $(69.2 \%)$. Mean duration of ICU stay and ventilation was $4.3 \pm 0.8$ and $2.9 \pm 0.9$ days, respectively. ICU admissions for asthma peaked during 1985 (53 patients) and a similar peak occurred in 1989 (52 admissions). Overall, there was no significant change in the average number of yearly admissions for asthma $(-0.4,95 \% \mathrm{CI}-1.44-0.61, \mathrm{p}=0.44)$ or the use of IPPV $(-0.1,95 \%$ CI $-0.87-0.65, p=0.78)$ which was required by a mean of $73.1 \pm 12.3 \%$ of patients per year (fig. 4). In-hospital mortality was $2.2 \%$ and there was no seasonal distribution of deaths.

\section{Discussion}

The results of this study suggest that there has been a reduction in the number of deaths from asthma in the Cape Town City Council area from 1980-1997, with the greatest reduction occurring in people $<55$ yrs of age. Consistent with studies from other countries, paediatric deaths accounted for a relatively small portion of the total asthma mortality $[8,9]$. The decline in asthma mortality is also consistent with recent trends in some countries [10-12]. However, this is the first study to describe a decline in asthma mortality in an African population.

A potential limitation of this study is the use of death certifications to investigate trends in mortality. Misclassification of the cause of death is an important potential source of error in studies of asthma mortality [13]. An attempt has been made to minimize this by including only death certificates for subjects in which asthma was listed as the sole cause of death or in association with respiratory failure. Restricting the analysis to these cases has been reported to decrease the calculated mortality by more than a third [3]. Furthermore, to obviate inaccuracies in attributing death to asthma as a result of diagnostic transfer from chronic obstructive pulmonary disease (COPD), data of those $<55$ yrs were analysed separately [14]. The reduction in asthma deaths found in people $<55$ yrs (in whom COPD is less likely to be a confounding factor) supports the observation that asthma mortality has truly declined.

Over the same period, ICU admissions for asthma were studied in order to investigate patients with NFA who may have died without such care. The number of paediatric asthma ICU admissions declined, while no change in adult admissions was demonstrated. Although ICU admission criteria may vary depending on the attending physician and the availability of beds, the ventilation rates for both paediatric and adult ICUs remained constant over the study period, indicating that there was no increase in the number of patients with NFA. The observed reduction in asthma mortality is therefore not attributable to increased utilization of ICU facilities. Although data from all ICU facilities in the reference area were not available for inclusion, the hospitals surveyed account for the majority of public beds in the area. It is unlikely that increased utilization of private ICU beds has occurred, as these are few and asthma admissions form a small proportion of their case load.

The mortality rate of patients admitted to the ICU $(2.2 \%)$ is similar to the overall in-hospital mortality reported in other studies $[15,16]$. Of greater concern is the large proportion of deaths at health facilities in general hospital wards or clinics, which accounted for one third of deaths in people $<55 \mathrm{yrs}$. Although $50 \%$ of such deaths occurred at health facilities without ICUs, emergency care and stabilization of patients prior to referral should be possible at primary and secondary care centres. However, a substantial number of patients may have been dead on arrival at the health facility, and these deaths wrongly recorded as in-hospital deaths. Nevertheless, possible reasons for failure of the health services need to be explored. Furthermore, a proportion of the remaining in-hospital deaths may have been avoided had suitable ICU facilities been available.

The majority of asthma deaths $(72.5 \%)$ occurred at home. Nonavailability of healthcare facilities coupled with poor transport and emergency services, inadequate home management of acute asthma or underrecognition of the severity of an attack, may be contributory factors $[17,18]$. Furthermore, poor socioeconomic status by limiting access to and affordability of health services may be an important reason for failure to obtain emergency treatment [19]. As evidence of this, the highest death rates occurred in ethnic groups from historically deprived social circumstances. The ethnic differences in asthma mortality are consistent with previous studies in this region $[3,4]$, and with studies from other countries in which ethnic factors and socioeconomic status have a modest effect on asthma prevalence, but a large effect on asthma hospitalization and mortality rates [19, 20]. Under an apartheid system, Black and mixed race people suffered discrimination and socioeconomic deprivation resulting in the provision of inferior health services compared to White patients. In addition, the higher mortality amongst mixed raced people may relate to environmental factors including smoking, as this urban population has the highest smoking prevalence among ethnic groups in South Africa [21].

Possible reasons for the decline in asthma mortality include improved access to healthcare, a greater emphasis on asthma education, wider use of inhaled corticosteroids and better recognition and treatment of acute asthma. However, the disparity in asthma mortality by ethnic group and the predominance of deaths occurring outside a health facility and on weekends suggest that economic factors and access to care remain important contributors to asthma mortality in Cape Town [17-20]. Although airway obstruction may evolve rapidly, the majority of asthma attacks develop over hours, allowing the opportunity for effective therapy to be instituted 
[22]. Future interventions to further reduce asthma mortality should focus on provision of quality healthcare and asthma education particularly amongst mixed race and Black populations.

\footnotetext{
Acknowledgements. The authors thank R. Raine for access to GSH ICU records, R. Blease of the Cape Town City Council for access to population and mortality data and F. Little and J. Irlam for statistical advice.
}

\section{References}

1. Sears MR. Changing patterns in asthma morbidity and mortality. J Investig Allergol Clin Immunol 1995; 5: $66-72$.

2. Molfino NA, Slutsky AS. Near-fatal asthma. Eur Respir J 1994; 7: 981 -990.

3. Benatar SR, Ainslie GM. Deaths from asthma in Cape Town 1980-1982. S Afr Med J 1986; 69: 669-671.

4. Ehrlich RI, Bourne DE. Asthma deaths among coloured and White South Africans: 1962 to 1988. Respir Med 1994; 88: 195-202.

5. South African Pulmonology Society Adult Asthma Working Group. Guidelines for the management of chronic asthma in adults - 2000 update. S Afr Med J 2000; 90: 536-548.

6. McFadden ER Jr, Warren EL. Observations on asthma mortality. Ann Intern Med 1997; 127: 142-147.

7. Annual Report of the Medical Officer of Health 1995/ 1996. City of Cape Town, Health Department ISSN $1021-4798$

8. Martin AJ, Campbell DA, Gluyas PA, et al. Characteristics of near-fatal asthma in childhood. Pediatr Pulmonol 1995; 20: 1-8.

9. Campbell DA, McLennan G, Coates JR, et al. A comparison of asthma deaths and near-fatal attacks in South Australia. Eur Respir J 1994; 7: 490-497.

10. Kemp T, Pearce N. The decline in asthma hospitalisations in persons $0-34$ years in New Zealand. Aust $N Z$ J Med 1977; 27: 578-581.

11. Campbell MJ, Cogman GR, Holgate ST, Johnston SL. Age specific trends in asthma mortality in England and Wales, 1983-95: results of an observational study. BMJ 1997; 314: 1439-1441.

12. Vergara C, Caraballo L. Asthma mortality in Columbia. Ann Allergy Asthma Immunol 1998; 80: 55-60.

13. Guite HF, Burney PG. Accuracy of recording of deaths from asthma in the UK: the false negative rate. Thorax 1996; 51: 924-928.

14. Reid DW, Hendrick VJ, Aitken TC, Berrill WT, Stenton SC, Hendrick DJ. Age-dependent inaccuracy of asthma death certification in Northern England, 1991 - 1992. Eur Respir J 1998; 12: 1079-1083.

15. Mountain RD, Sahn SA. Clinical features and outcome in patients with acute asthma presenting with hypercapnia. Am Rev Respir Dis 1988; 138: 535-539.

16. Richards GN, Kolbe J, Fenwick J, Rea HH. Demographic characteristics of patients with severe life threatening asthma: comparison with asthma deaths. Thorax 1993; 48: 1105-1109.

17. Jones AP, Bentham G. Health service accessibility and deaths from asthma in 401 local authority districts in England and Wales, 1988-1992. Thorax 1997; 52: 218-222.

18. Kaliner MA. Asthma deaths: A social or medical problem? JAMA 1993; 269: 1994-1995.

19. Marder D, Targonski P, Orris P, Persky V, Addington W. Effect of racial and socio-economic factors on asthma mortality in Chicago. Chest 1992; 101: Suppl. 6. S426-S429.

20. Ng TP, Tan WC. Temporal trends and ethnic variations in asthma mortality in Singapore, 19761995. Thorax 1999; 54: 990-994.

21. Reddy P, Meyer-Weitz A, Yach D, Van der Burgh C. Smoking status, knowledge of health effects and attitudes towards tobacco control in South Africa. $S$ Afr Med J 1996; 86: 1389-1393.

22. Kolbe J, Fergusson W, Garrett J. Rapid onset asthma; a severe but uncommon manifestation. Thorax 1998; 53: $241-247$. 